Voix et Images

\title{
Françoise et Madeleine, pionnières du journalisme féminin au Québec
}

\section{Aurélien Boivin et Kenneth Landry}

Volume 4, numéro 2, décembre 1978

Guy Lafond

URI : https://id.erudit.org/iderudit/200154ar

DOI : https://doi.org/10.7202/200154ar

Aller au sommaire du numéro

Éditeur(s)

Les Presses de l'Université du Québec

ISSN

0318-9201 (imprimé)

1705-933X (numérique)

Découvrir la revue

Citer cet article

Boivin, A. \& Landry, K. (1978). Françoise et Madeleine, pionnières du journalisme féminin au Québec. Voix et Images, 4(2), 233-243.

https://doi.org/10.7202/200154ar d'utilisation que vous pouvez consulter en ligne.

https://apropos.erudit.org/fr/usagers/politique-dutilisation/ 


\title{
Françoise et Madeleine, pionnières du journalisme féminin au Québec
}

\begin{abstract}
Adam était le pouvoir exécutif, Ėve le pouvoir délibératif. Or, gouverner, c'est prévoir, suivant un axiome moderne. Adam n'a rien prévu. II était indigne du pouvoir. Donc, le sceptre doit incontestablement passer à Eve.
\end{abstract}

(Françoise, Chroniques du lundi, p. 20.)

La femme québécoise a mis du temps à gagner ses lettres de noblesse tant en littérature qu'en journalisme. II faut attendre les années 1880 pour qu'enfin se manifeste la première romancière, une romancière de grand talent d'ailleurs, Laure Conan, et les années 1890 pour que se fassent entendre les premières femmes journalistes qui n'ont, comme la première romancière, d'autre choix que de se cacher sous un pseudonyme. C'est Françoise d'abord, née Robertine Barry, qui, dès septembre 1891, signe dans la Patrie libérale d'Honoré Beaugrand des chroniques qu'elle réunira plus tard sous le titre Chroniques du lundi. Puis, à peu près en même temps, surgissent d'autres femmes qui, comme Françoise, ont décidé de travailler à l'épanouissement de la femme, à l'amélioration de sa condition: Josephte (née Joséphine Marchand, épouse du sénateur Raoul Dandurand), Madeleine (née Anne-Marie Gleason, épouse de Wilfrid-A. Huguenin), Gaétane de Montreuil (née Georgine Bélanger, épouse du poète Charles Gill), Fadette (née Henriette Dessaules), Colombine (née Éva Circé, épouse de Salomon Côté), Colette (née Édouardine Lesage), Ginevra (née Georgiana Lefebvre) et combien d'autres qui ont pris la relève et poursuivi les mêmes buts.

II n'est pas dans notre intention de présenter à tour de rôle, dans le cadre restreint de cet exposé, chacune de ces grandes dames qui ont eu leur mot à dire dans la longue marche de la femme vers son émancipation; nous nous contenterons de faire connaître, trop rapidement hélas!, deux journalistes qui ont profondément marqué la lutte des femmes à la fin du siècle dernier et au début du $X X^{e}$ : Françoise, parce qu'elle fut la première femme à embrasser cette carrière et à se servir de sa plume pour défendre et améliorer la condition des femmes au Québec, et Madeleine, parce qu'elle a donné un nouvel essor aux aspirations de la première et parce que l'œurre qu'elle nous a laissée est très abondante. 


\section{Françoise}

Née à L'îsle-Verte, comté de Témiscouata, en 1863, d'un père irlandais et d'une mère canadienne-française, Robertine Barry débarque à Montréal en 1891, après de solides études au couvent de Trois-Pistoles et celui des Ursulines de Québec. Elle devient rédactrice de la Patrie et prend la direction d'une page littéraire qui eut ses moments de gloire et de fortune, "le Coin de Fanchette». Déléguée du gouvernement canadien à l'Exposition universelle de Paris en 1900, puis à celle de Milan en 1906, elle fonde en 1902, le Journal de Françoise, revue féminine bimensuelle qui disparaît en avril 1909. Cette année-là, la journaliste devient inspectrice du travail féminin dans les établissements industriels; fonction qu'elle occupe peu longtemps puisqu'elle tombe malade. Elle meurt en janvier 1910. Elle avait publié en 1895, un recueil de contes et nouvelles: Fleurs champêtres, puis, probablement en 1896, Chroniques du lundi. En 1905, elle avait fait représenter une pièce de théâtre : Méprise.

Dans ses chroniques journalistiques, Françoise aborde différents sujets : religieux, social, politique, économique... Elle donne son opinion, conseille, désavoue, condamne, dans une langue toujours soignée qui témoigne d'une grande culture et d'un grand amour pour la langue française. Nous traiterons surtout des sujets qui s'intéressent à la femme.

Comme elle le fait dans "Superstitions», un conte de Fleurs champêtres, Françoise dénonce souvent, dans ses chroniques, les nombreuses superstitions qui ont prise sur les femmes de sa génération. Par exemple, une femme est assurée de gagner à la loterie si la première personne qu'elle rencontre, après s'être procurée son billet, est un bossu. Ce bossu doit évidemment être un mâle car, comme tout le monde le sait, «la rencontre d'une bossue est malchanceuse aux personnes de son sexe, comme elle est heureuse aux autres. Et vice-versa". Si une femme rencontre, par hasard, l'une de ces disgraciées de la nature, elle doit «cracher par terre du côté opposé à celui où elle aura passé ${ }^{\prime}$. Toutefois, même si elle suit à la lettre cette recette magique, la femme que nous présente Françoise doit se contenter d'un bien modeste prix de $\$ 0,10$ à la loterie.

La journaliste de la Patrie démythifie encore les guérisseuses" ${ }^{2}$ " ces supposées thaumaturges" qui ne guérissent rien sauf la curiosité et le porte-monnaie. Elle met en garde la population féminine contre ce genre d'exploitation. Elle n'est pas sans se rendre compte que la femme de la fin du $X I X^{e}$ siècle est peu instruite et bien mal préparée à sa vie de mère et d'épouse.

Pour la mieux préparer à son rôle de femme, Françoise prône la mise sur pied d'écoles ménagères ${ }^{3}$ dans toutes les régions du Québec. Dans ces écoles, outre l'art culinaire et l'art de tenir une maison, y sont enseignés: la couture, l'économie domestique, l'hygiène, les premiers soins, la chimie alimentaire, la comptabilité domestique, l'anatomie, la formation physique et morale de l'enfant et, bien entendu, l'instruction religieuse. Toutefois, 
dans l'esprit de Françoise, de telles écoles, qui allaient disparaître avec la réforme scolaire des années 1960 , répondent bien à la mission de la femme: l'éducation des enfants et la tenue de la maison. Mais ce n'est pas là, selon elle, l'unique mission de la femme canadienne-française. Françoise approuve sans équivoque - et elle fut sans doute la première au Québec - la décision de nombre de femmes et de jeunes filles de ne plus vivre aux crochets de leur époux ou de leur famille et de quitter le foyer pour gagner elle-même leur vie. Car, même si elle prétend n'avoir guère de voix au conseil, Françoise trouve tout à fait inadmissible qu'une épouse, par exemple, doive «cajoler, entortiller un homme, lui couler du beurre dans le dos, comme on dit vulgairement, pour obtenir par ces petits moyens un objet de luxe que la fantaisie convoite ". Mais elle n'est pas sans craindre quelque peu ce mouvement d'indépendance qui semble déjà irréversible. Ces femmes, ces jeunes filles qui ont fréquenté des couvents dirigés par des religieuses ne sont pas préparées adéquatement pour affronter les dangers qui les guettent quand elles auront rejoint le marché du travail. Mal préparées, elles le sont d'abord intellectuellement. Aussi Françoise exige-t-elle - et là encore, elle est la première à le faire au Québec - que soient mis au programme, dans les couvents et les écoles privées pour jeunes filles, des cours de dactylographie, de sténographie et de tenue de livres. Mal préparées, elles le sont aussi moralement. Car, "c'est encore moins au couvent que le[s] jeune[s] fille[s] apprendr[ont] les grandes leçons pratiques de la vie». Selon la journaliste, les religieuses devront donc surmonter "leur antipathie naturelle à l'égard des hommes et présenter à leurs élèves leur futur patron «non comme un monstre", mais... comme un "ennemi naturel qu'il faut [leur] apprendre à redouter". A la jeune fille fin du siècle, il faut donc lui enseigner ale grand souci de sa dignité de femme, l'influence salutaire qu'elle peut exercer en tant que femme", peu importe son occupation, sa position; que "l'homme, - le patron surtout, - n'est pas toujours un protecteur"; qu'elle doit «se défier d'elle-même, de son pauvre cœur de femme trop prompt à s'attacher, trop prêt à se donner»; que c'est d'elle et non de l'homme "que doit venir la résistance aux tentations les plus belles comme les plus terribles". Françoise est tout à fait convaincue qu' «il ne tiendra qu'à la femme de faire régner, où elle travaillera, une atmosphère pure et sai$n e^{5}$ ". Voilà une préoccupation de l'époque, voilà une des fréquentes recommandations que Françoise ne cesse de répéter surtout aux jeunes filles qui quittent le couvent pour entrer dans un bureau ou dans une manufacture.

Consciente du rôle que doit jouer la femme dans la société, de la place qui lui revient et qu'elle doit occuper, Françoise réclame, pour la femme, l'enseignement du latin 6 , l'éducation universitaire et l'accès aux professions libérales'. Elle favorise encore l'enseignement de l'éducation physique, si négligé dans les écoles de filles de langue française, et insiste sur la nécessité de professeurs compétents: "Je connais des couvents, écrit-elle, où ce sont des religieuses elles-mêmes qui donnent les leçons. 
On peut aisément se figurer que le voile, le bonnet, la guimpe et tout l'habit de la maîtresse ne lui permettent pas de donner la leçon d'une façon commode ${ }^{8}$." Elle se montre favorable à l'adoption d'un costume spécial pour faciliter les mouvements lors des exercices physiques ${ }^{9}$, ce qui lui aura sans doute valu des réprimandes de la part de membres influents du clergé...

Elle loue encore le travail honorable mais combien pénible des institutrices, mal rémunérées et souvent obligées ad'acheter le combustible nécessaire à leur école ». Pour Françoise, ces femmes dévouées, ces servantes, sont vraiment à plaindre. Comme l'a écrit Madeleine, sa collègue, "c'est une honte pour [la] province de Québec [...] de rabaisser bien en bas du travail mécanique, l'œuvre d'intelligence et de cœur de ces pauvres dévouées, de ces obscures missionnaires qui prêchent la science et qui crèvent de froid et de misère ${ }^{10}$ ". Voilà un système déplorable qu'il faudra changer à brève échéance pour favoriser l'instruction et inciter les jeunes filles à choisir cette noble profession.

Dans son souci constant d'instruire la femme, d'aiguiser son sens social, d'élever son niveau intellectuel, Françoise encourage la création d'un ministère de l'Instruction publique ${ }^{11}$ et l'éclosion de bibliothèques publiques. Elle part en guerre contre les autorités municipales qui retardent le projet de construction de la Bibliothèque municipale de Montréal ${ }^{12}$. C'est elle qui entreprend la campagne pour la création d'une bibliothèque à Beauharnois $^{13}$ et à Saint-Jean-lberville ${ }^{14}$ et d'une section de livres français à la bibliothèque de Waterloo' ${ }^{15}$. Elle écrit : “le livre sera, chez nous» dans ce pays jeune, "un puissant facteur pour son éducation comme pour son instruction». D'où l'importance, “de doter nos paroisses, nos villages de bibliothèques qui exerceront [...] une action bienfaisante et civilisatri$c e^{16}$ ». Si la journaliste ne réclame pas le droit de vote, elle exige, en revanche, plus de facilité pour les femmes locataires et propriétaires qui peuvent exercer leur droit aux élections municipales. Comme "les bureaux de votation (polls) sont généralement placés dans des endroits où les femmes ne peuvent se présenter sans s'exposer à une promiscuité désagréable», Françoise réclame “des bureaux de vote exclusivement consacrés aux femmes". Et, consciente de sa mission, elle ne manque pas de reproduire dans son journal, quelques jours avant les élections municipales, les textes de loi autorisant les femmes locataires et propriétaires à voter. Elle les incite ainsi à se prévaloir de leur droit ${ }^{17}$.

Elle recommande encore aux mères de famille «de développer, comme une autre religion, dans l'esprit des petits enfants, [l']amour de la patrie, ce sentiment de patriotisme éclairé, destiné à produire tant de bien sur un peuple ${ }^{18} "$. Comme les mères sont les éducatrices des enfants, c'est à elles qu'incombe la tâche de leur enseigner "l'amour de Dieu et celui de la patrie» et de leur inculquer, «dès le premier âge, les principes fondamentaux de l'honneur et du devoir pour tout homme de se dévouer à la cause commune ${ }^{19}$ ". Elle dénonce ce monstre qu'on appelle alcoolisme, 
prend la défense des épouses des alcooliques, réclame la diminution des buvettes dans le grand Montréal et la multiplication des boulangeries ${ }^{20}$ afin de donner du pain à tout le monde, aux pauvres et aux deshérités pour lesquels Françoise n'a jamais cessé de réclamer justice. Tout au long de sa carrière de femme de lettres, Françoise a cru en l'égalité pour tous, en une justice sociale, surtout pour les femmes qu'elle considère souvent comme des êtres exploités. Toute sa vie, elle a donc appuyé toute initiative susceptible d'épanouir la femme et d'améliorer le sort des malheureux, des miséreux, des infirmes, des défavorisés. Elle encourage, par exemple, les bazars, kermesses, concerts, pageants, autant de manifestations sociales dont les profits servent à diminuer la misère et la pauvreté21. Elle soutient les efforts des responsables du mouvement réclamant "une maison de refuge, entretenue par l'État, pour les infirmes et les miséreux ${ }^{22}$ ", d'un hospice pour les aveugles. Elle approuve la fondation de l'Association de couture, une cuvre "destinée à venir en aide à nos institutions de charité dans le soin et l'entretien des pauvres ${ }^{23}$.

Elle encourage les bénévoles qui se donnent sans compter pour rendre l'enfance heureuse ${ }^{24}$. Elle compatie au sort réservé aux vendeuses de magasins et plaide avec énergie pour que ces employées obtiennent des sièges ${ }^{25}$. Elle applaudit à l'annonce de la fondation de la Société protectrice pour les femmes, les enfants et les vieillards ${ }^{26}$ et se réjouit de la construction d'un asile destiné à recueillir et à soigner d'abord "les pauvres femmes que le sort et les maris, plus encore, ont inhumainement traitées ${ }^{27}$ ", et ensuite les enfants et les vieillards abandonnés. Françoise, en outre, ne rate jamais une occasion pour conseiller les femmes sur différents sujets qui les intéressent et pour leur enseigner l'étiquette ${ }^{28}$ : elle insiste, par exemple, sur la ponctualité, la tenue à table, au salon... Elle ne se gêne pas, à l'occasion, pour condamner l'attitude de certains hommes, leurs gaucheries, leur impolitesse et leur manque de savoir-vivre. Elle réprimande ceux «qui ne cessent de chialer leurs femmes quand elles veulent renouveler une toilette ou un simple morceau de linge ${ }^{29}$ ". Enfin, convaincue de l'importance de plus en plus grande de la femme, elle propose l'admission des femmes à l'Académie ${ }^{30}$ que veut fonder le docteur Ernest Choquette, romancier et homme de théâtre fort populaire au début du siècle, et à la Société royale du Canada ${ }^{31}$. Mais il faudra attendre encore plusieurs années avant que cet honneur échoie à une femme. Gabrielle Roy deviendra la première femme à pénétrer cette chasse gardée masculine de la Société royale. D'autres l'imitent et les femmes auront enfin accès à l'Académie canadienne-française.

\section{Madeleine}

Comme sa collègue Françoise, Madeleine est née d'un père irlandais et d'une mère canadienne-française. De son vrai nom Anne-Marie Gleason, elle voit le jour à Rimouski en 1875. Elle fait ses études au couvent des sceurs de la Charité à la Malbaie, puis à Rimouski. Elle collabore au Mon- 
de illustré et au Journal de Rimouski avant de se rendre à Ottawa où elle devient chroniqueuse au journal le Temps. En 1901, Israël Tarte lui confie la page féminine de la Patrie. Elle remplace Françoise, qui avait démissionné pour fonder son propre journal. A la Patrie, sous le pseudonyme de Madeleine, elle inaugure le "Royaume des femmes", qu'elle dirige pendant dix-neuf ans. En 1913, elle préside à la fondation de la Bonne Parole, organe de la Fédération nationale Saint-Jean-Baptiste. En 1919, elle met sur pied la Revue moderne, l'ancêtre de Chattelaine. Son cuvre littéraire comprend plus de trois mille ${ }^{32}$ chroniques, billets, contes, lettres, conférences, causeries et articles de tous genres, publiés entre 1897 et 1943, année de sa mort. Sous les titres suivants, elle avait réuni en volume ses meilleurs articles: Premier Péché, 1902; le Long du chemin, 1912 et le Meilleur de soi, 1924. Elle a aussi publié deux pièces de théâtre et un recueil de notices biographiques intitulé : Portraits de femmes, 1938. Elle avait épousé en 1904 le docteur Wilfrid-A. Huguenin.

Qualifiée par Camille Roy de "reine incontestée au royaume de la chronique féminine ${ }^{33}$ ", Madeleine a travaillé toute sa vie à l'amélioration de la condition des femmes de son époque. Dans les colonnes de la Patrie, de la Bonne Parole et de la Revue moderne, ses billets s'inspirent de la vie quotidienne montréalaise. La journaliste veut effectuer une sorte d'évangélisation des masses féminines. Elle prêche les bonnes lectures, la tempérance, le soutien des œuvres de charité, la diligence au travail, le patriotisme - en un mot, les causes "justes". De plus, elle ne prêche pas dans le désert, si l'on se fie à sa volumineuse correspondance. Son public l'oblige à aborder fréquemment des questions touchant la vie du cœur. Elle publie alors un grand nombre de récits attendrissants et moralisateurs, dans un style qui frôle parfois la mièvrerie ${ }^{34}$ et dans le but avoué de défendre les vertus chrétiennes traditionnelles. Dans son cuvre, Madeleine est moraliste. Mais sa morale n'a rien de théorique, puisqu'elle se traduit par d'innombrables conseils pratiques tout au long de ses chroniques. En littérature, par exemple, elle s'oppose aux lectures frivoles des jeunes filles de son époque ${ }^{35}$. Voulant combattre l'indifférence du public à l'égard de la production littéraire québécoise, elle signale, dans ses nombreuses recensions, les livres qui méritent d'être lus et «ceux qui ne se gênent pas d'être ridicules ${ }^{36}$ ". A ses collègues, les critiques littéraires, qui seraient tentés de louanger des œuvres ratées, elle adresse le reproche: «Moins d'encens et plus de bon sens ${ }^{37}$. "Elle s'insurge contre l'attitude "Hors le terroir, pas de talent ${ }^{38}$ " des tenants d'un régionalisme littéraire exclusif. "L'indigénisme est un leurre ${ }^{39}$ ", dira-t-elle en prenant la défense de la liberté de l'écrivain d'ici de "réfléter en français l'âme de la race ${ }^{40}$ " sans subir les contraintes d'un programme ou d'une école de pensée.

La langue française tient une place toute spéciale dans l'œuvre de Madeleine. Elle appuie les mouvements de revendication des droits de la langue française au Québec ${ }^{41}$ et elle expose son point de vue au premier Congrès de la langue française en 1912 dans une conférence intitulée "le Foyer gardien de la langue française ". 
Que la mère reste la gardienne de ce foyer où doit se conserver la langue française! Elle en est la grande prêtresse et c'est à elle qu'incombera toujours le soin de cultiver, au cœur de l'enfant, le respect et l'amour de ses origines, le culte de la langue...42

Ces propos ne sont pas féministes, du moins pas dans leur esprit. Madeleine se défendait bien d'être féministe, car à l'époque, le mouvement était tenu en suspicion par les tenants de l'idéologie officielle. En 1918, monseigneur Louis-Adolphe Paquet de l'université Laval voyait dans le féminisme "un mouvement pervers, une ambition fallacieuse [qui] entraîne hors de sa voie la plus élégante moitié de notre espèce, et menace les bases mêmes de la famille et de la société ${ }^{43}$ m. Claude-Henri Grignon et Henri Bourassa iront jusqu'à caractériser de "bas-bleu ${ }^{44}$ " et de «femmeshommes ${ }^{45}$ " celles qui revendiquent l'extension du droit des femmes dans la société. Madeleine essaie de se tenir loin des grands débats sur cette question. Elle préfère inciter ses lectrices à améliorer leur condition dans des domaines précis comme celui de l'éducation, de la politique, du travail et de la famille. Elle voudrait obtenir une meilleure instruction pour la jeune fille, certains droits pour la citoyenne, du travail assuré et rémunérateur pour l'ouvrière, de l'équité et du respect pour la femme mariée, de la sécurité et de l'aide pour la mère au foyer.

Sans négliger la culture intellectuelle, elle insiste sur l'importance d'enseigner aux jeunes filles un peu plus d'économie domestique. «ll ne faut pas élever les filles pour en faire des marquises ou des duchesses, mais les mettre en état de tenir la maison... préparons la jeune fille à la lutte pour la vie, afin qu'elle puisse combattre victorieusement pour le bienêtre de la famille, dont elle deviendra le soutien et l'égide ${ }^{46}$ !"

Ce sens pratique, voire pragmatique, traverse toute l'œuvre de la chroniqueuse. Sur la question du droit de vote pour les femmes, qui a fait couler beaucoup d'encre au début du siècle, Madeleine l'approuve en principe, mais elle rappelle aux politiciens "que nous sommes une poignée et que nous ayons le devoir strict d'être là quand il s'agit de défendre notre langue... d'être là pour empêcher la passation des lois qui nous étouffent, d'être là pour affirmer nos droits à la vie ${ }^{47} \%$. Elle ne se cache pas, cependant, que la femme, n'ayant pas reçu d'éducation politique, sera sans doute exploitée lors des élections ${ }^{48}$.

Quant au droit de la femme au travail, Madeleine prend prétexte du refus du gouvernement provincial de permettre aux femmes de pratiquer la sténographie au Palais de Justice pour démystifier l'attitude des hommes à l'égard de l'ouvrière salariée ${ }^{49}$. Elle conclut : “lci, où tout est à faire, la loi d'un travail universel s'impose ${ }^{50}$."

Ainsi, la jeune fille, la citoyenne et l'ouvrière ont donc droit à tous les égards de la chroniqueuse. Pourtant, c'est la mère au foyer qui se mérite les plus belles épithètes: cet «ange gardien de la famille», cet «être suave et doux qui se penche sur les berceaux ${ }^{51}$ " incarne, aux yeux de Ma- 
deleine, l'âme de la race. Elle perçoit comme un devoir supérieur son rôle de "consolider, de grandir, d'exhausser la vie familiale... d'assurer la paix et la joie des familles, de supprimer les inutiles souffrances, de soulager les détresses morales comme les déchéances physiques, et de préparer la génération qui pousse à de plus hautes et plus saines destinées ${ }^{52}$ ». C'est tout un programme!

En 1921, lors d'une conférence enflammée sur la solidarité féminine prononcée devant la Fédération nationale de la Société Saint-Jean-Baptiste de Montréal, Madeleine dresse le bilan de l'évolution de la femme québécoise. Selon elle, la guerre de 1914-1918 a consacré l'émancipation de la femme. La conférencière reproche aux femmes d'ici d'être des esclaves qui aiment leurs chaînes. Elle les incite alors à se débarrasser de leur prétendue infériorité intellectuelle et morale en se joignant au mouvement de solidarité en voie de création. Voulant réconcilier la femme émancipée et la mère de famille, elle déclare à son auditoire : «[...] il faut prouver que la femme consciente de sa valeur, capable d'aider l'homme dans ses travaux les plus sérieux, est aussi la mère idéale qui saura élever des fils à l'esprit libre, sincère et généreux, des fils dignes de la race canadienne-françai$\mathrm{se}^{53} \mathrm{~m}$.

De telles déclarations en disent long sur la conception qu'on se faisait de la femme québécoise au début du vingtième siècle. Par le biais d'une cuvre journalistique imposante, Françoise et Madeleine ont voulu tout simplement rappeler au public lecteur l'importance du point de vue féminin. II n'est pas question à cette époque de supplanter l'homme. La plupart des femmes journalistes, selon Réginald Hamel, auteur d'une étude sur Gaétane de Montreuil, «pratiquent ce conservatisme tendrement agressif qui incline vers les premières conquêtes du féminisme, mais qui refuse encore de rompre avec les traditions sociales ${ }^{54}$ m.

Ces pionnières du journalisme féminin ont compris que le rôle de la femme ne pouvait être renversé ou modifié en profondeur sans que l'édifice humain tout entier en soit bouleversé.

En terminant, il serait peut-être utile de noter que l'on est encore loin d'avoir analysé l'ampleur de la contribution du journalisme féminin à l'histoire littéraire et culturelle du Québec. Nos femmes de lettres n'ont encore fait l'objet d'aucune étude d'ensemble, exception faite de "brèves apologies ${ }^{5}$ " publiées en 1920 et d'une thèse ${ }^{56}$ soutenue en 1947 . Le temps est venu de réévaluer l'apport des femmes à la littérature québécoise, mais il faudra dépouiller systématiquement la presse périodique pour y arriver.

Aurélien Boivin et Kenneth Landry (Dictionnaire des œuvres littéraires du Québec) 
1. Chroniques du lundi, [Montréal, s.é., 1896?], p. 37-40.

2. Ibid., p. 5-10.

3. "L'École ménagère", le Journal de Françoise, vol. VII, $n^{\circ} 11$ (29 août 1908), p. 166-167. Nous utiliserons les lettres $J$. de $F$. pour désigner dorénavant ce périodique.

4. Chroniques du lundi, p. 13.

5. "Les Jeunes Filles dans les bureaux», J. de F., vol. I, n²3 (21 février 1903), p. 269-270.

6. Chroniques du lundi, p. 125-128.

7. Ibid., p. 305-309.

8. Ibid., p. 195.

9. Ibid., p. 196-197.

10. «Le Congrès féminin », J. de F., vol. VI, n 6 (15 juin 1907), p. 89-90.

11. «A propos d'un Ministère de l'Instruction publique», J. de F., vol. VI, $n^{\circ} 13$ (5 octobre 1907), p. 198.

12. "A propos de bibliothèques", J. de F., vol. I, $n^{\circ} 15$ (1902), p. 169-170.

13. "La Bibliothèque de Beauharnois », J. de F., vol. V, n 4 (15 mai 1906), p. 55-56.

14. "L'CEuvre des bibliothèques", J. de F., vol. IV, n² 21 (février 1906), p. 330-331.

15. Ibid

16. "La Bibliothèque de Beauharnois ", J. de F., vol. V, $n^{\circ} 4$ (15 mai 1906), p. 55.

17. "Élections municipales", J. de F., vol. II, n०14 (17 octobre 1903), p. 192.

18. Chroniques du lundi, p. 222.

19. "Femmes, soyons patriotes!", J. de F., vol. II, $n^{\circ} 6$ (20 juin 1903), p. 82.

20. "Ligue antialcoolique", J. de $F$. , vol. VII, $n^{\circ} 1$ (4 avril 1908), p. 2.

21. Chroniques du lundi, p. 202.

22. Ibid., p. 128.

23. "L'Association de couture", J. de F., vol. IV, $n^{\circ} 1$ (1 ${ }^{\mathrm{er}}$ avril 1905), p. 2.

24. Chroniques du lundi, p. 102.

25. Ibid., p. 132.

26. "Une couvre recommandable», J. de F., vol. II, $n^{\circ} 15$ (7 novembre 1903), p. 195.

27. Ibid.

28. Chroniques du lundi, p. 183-185.

29. Ibid., p. 12-15.

30. “Mal d'académie », J. de $F_{\text {, }}$ vol. VII, $n^{\circ} 21$ (6 février 1909), p. 333.

31. "La Société royale», J. de F., vol. VII, $n^{\circ} 21$ (6 février 1909), p. 333.

31. "La Société royale", J. de F., vol. VI, n 3 (4 mai 1907), p. 38.

32. Maria Eugénia de Matos-Andrade, «Biographie et bibliographie descriptive de Madeleine (1875-1943)", thèse de D.E.S., Montréal, Université de Montréal, 1970,488 [3] f.

33. Camille Roy, Érables en fleurs, Québec, Imprimerie de l'Action sociale, limitée, 1923, p. 180.

34. Sœur M. Eleanor, «les Écrivains féminins du Canada français de 1900 à 1940 ", thèse de maîtrise ès arts, Québec, Université Laval, 1947, f. 61.

35. Myrto [pseudonyme d'Anne-Marie Gleason], "Chronique ", le Nationaliste, 21 janvier 1905, p. 3.

36. Madeleine, "Chronique ", la Patrie, 26 janvier 1903, p. 4.

37. Ibid., 9 janvier 1904, p. 22.

38. Madeleine, “la Liberté littéraire», la Revue moderne, 15 mars 1920, p. 7.

39. Ibid.

40. Ibid.

41. Madeleine, “On veut parler français », la Patrie, 15 mars 1902, p. 22.

42. Id., "le Foyer, gardien de la langue française", dans Premier Congrès de la langue française au Canada. Quêbec, 24-30 juin 1912. Mémoires, Québec, Imprimerie de l'Action sociale limitée, 1913, p. 536.

43. Louis-Adolphe Paquet, «le Féminisme ", le Canada français, décembre 1918 , p. 233. 
44. Claude-Henri Grignon [pseudonyme : VALDOMBRE], «Faits et Gestes. Le bas bleu sous l'orage", Pamphlets de Valdombre, novembre-décembre 1940 , p. 203-225.

45. Henri Bourassa, Femmes-Hommes ou hommes et femmes? Études à batons rompus sur le féminisme, Montréal, Imprimerie du Devoir, 1925, 83 [1] p.

46. Myrto, "Causerie. Les programmes d'études des écoles de filles", le Nationaliste, 19 mai 1905, p. 3.

47. Madeleine, "Chronique", /a Patrie, 15 avril 1918, p. 4.

48. Id., "Dans la mêlée", la Revue moderne, octobre 1921, p. 9-10.

49. Id., "Chronique», la Patrie, 3 mars 1902, p. 4.

50. Ibid.

51. Madeleine, “Entre nous", la Bonne Parole, mai 1918, p. 1.

52. Id., "la Solidarité féminine ", la Revue moderne, 15 mai 1921, p. 49.

53. Ibid., p. 50.

54. Réginald Hamel, Gaëtane de Montreuil, [Montréal], L'Aurore. [1976], p. 73.

55. Georges Bellerive, Brèves Apologies de nos auteurs féminins, Québec, Librairie Garneau, 1920, 137 [2] p.

56. Scour M. Eleanor, op. cit.

\section{BIBLIOGRAPHIE}

\section{Françoise}

\section{1-CEuvres:}

Fleurs champêtres, Montréal, la Cie d'Imprimerie Desaulniers, 1895, 205 p.

Chroniques du lundi, [Montréal, s.é., 1896], 325 p.

"Méprise. Comédie en un acte", représentée le 7 novembre 1905 à la salle Karn,

à l'occasion d'une soirée en l'honneur de Françoise.

II-Études :

BOIVIN, Aurélien, le Conte littéraire québécois au $\mathrm{XIX}^{\ominus}$ siècle. Essai de bibliographie critique et analytique. Préface de Maurice Lemire, Montréal, Fides, 1975, p. 43-51.

Robertine Barry) ", dans Dictionnaire des cuvres littéraires du Québec, t. I, Montréal, Fides, 1977, p. 268-270.

BRODEUR, Madame Donat (pseudonyme: Louyse de Bienville), "A Françoise”, dans Figures et Paysages. Préface d'Édouard Montpetit, Montréal, Beauche$\min , 1931$, p. 123-127; port.

CHASSE, Gertrude, "Bio-bibliographie de Françoise (Mlle Robertine Barry)". Préface de Colette Lepage. Montréal, École de bibliothécaires, Université de Montréal, 1945, x,67 f.

TURGEON, Louise, née Léonide Ferland (pseudonyme: Renée des Ormes), Robertine Barry. En littérature: Françoise. Pionnière du journalisme féminin au Canada. 1863-1910, Québec, [s.é.], 1949, 159 p.

\section{Madeleine}

\section{I-CEuvres :}

Premier Péché. Recueil de nouvelles et chroniques et d'une pièce de theátre en 1 acte [intitulée: l'Adieu du poète], Montréal, Imprimerie de la Patrie, $1902,162 \mathrm{p}$. 
Le Long du chemin, Montréal, Imprimerie de la Patrie, 1912, xv, $248 \mathrm{p}$.

En pleine gloire! Pièce en un acte, Montréal, Compagnie de publication de la Patrie limitée, 1919, 24 p.

Le Meilleur de soi, Montréal, éditions de la Revue moderne, 1924, 166 p. Illustrations de Robert Mathias.

Portraits de femmes, Montréal, Éditions de la Patrie, 1938, 188 p. III. [réimprimé la même année].

II -Études :

BÉLANGER, Georgine (pseudonyme: Gaëtane de Montreuil), “Examen de conscience ", la Presse, 10 janvier 1903, p. 17 :

ÉTHIER, Mireille, “Bio-bibliographie de Madeleine», Montréal, École de bibliothécaires, Université de Montréal, 1945, xxvii, $136 \mathrm{f}$.

GRIGNON, Claude-Henri (pseudonyme: Valdombre), «Faits et Gestes. Le bas-bleu sous l'orage ", Pamphlets de Valdombre, novembre-décembre 1940, p. 203-225.

MATOS-ANDRADE, Maria Eugénia de, «Biographie et bibliographie descriptive de Madeleine (1875-1943) ", thèse de D.E.S., Montréal, Université de Montréal, 1970,488 [3] f.

PLANTE, Juliette, "Madeleine, journaliste", thèse de maîtrise ès arts, Ottawa, Université d'Ottawa, 1962, xv, $121 \mathrm{f}$.

ROY, Camille, “Causerie littéraire. Madeleine", la Nouvelle-France, février 1905, p. 58-75 [reproduit dans Essais sur la littérature canadienne, 1907, p. 171195].

- "Le long du chemin, par Madeleine», dans Érables en fleurs. Pages de critique littéraire, Québec, [s.é.], 1923, p. 179-185.

\section{QUELQUES ÉTUDES SUR LE MOUVEMENT FÉMININ :}

BELLERIVE, Georges, Brèves Apologies de nos auteurs féminins, Québec, Librairie Garneau, 1920, 137 [2] p.

CONSEIL NATIONAL DES FEMMES DU CANADA, les Femmes du Canada, leur vie et leurs œuvres, Montréal, [s.é.], 1900, xiv, 474 p.

BOURASSA, Henri, Femmes-Hommes ou hommes et femmes? Études à battons rompus sur le féminisme, Montréal, Imprimerie du Devoir, 1925, 83 [1] p.

ELEANOR, M. (sœur), "les Écrivains féminins du Canada français de 1900 à 1940 ", thèse de maîtrise ès arts, Québec, université Laval, 1947, 214 [1] f.

HAMEL, Réginald, Bibliographie sommaire sur l'histoire de l'écriture féminine au Canada, 1769-1961, [Montréal], Université de Montréal, 1974, $134 \mathrm{p}$.

_. Gaëtane de Montreuil, [Montréal], L'Aurore, "Connaissance des pays québécois ", [1976], 205[7]p. 